\title{
Matching by Tone Mapping: Photometric Invariant Template Matching
}

\author{
Yacov Hel-Or, Member, IEEE, Hagit Hel-Or, Member, IEEE, and Eyal David,
}

\begin{abstract}
A fast pattern matching scheme termed Matching by Tone Mapping (MTM) is introduced which allows matching under non-linear tone mappings. We show that, when tone mapping is approximated by a piecewise constant/linear function, a fast computational scheme is possible requiring computational time similar to the fast implementation of Normalized Cross Correlation (NCC). In fact, the MTM measure can be viewed as a generalization of the NCC for non-linear mappings and actually reduces to NCC when mappings are restricted to be linear. We empirically show that the MTM is highly discriminative and robust to noise with comparable performance capability to that of the well performing Mutual Information, but on par with NCC in terms of computation time.
\end{abstract}

Index Terms-Pattern matching, template matching, structural similarity, photometric invariance, Matching by Tone Mapping, MTM, nonlinear tone mapping.

\section{INTRODUCTION}

$\mathrm{T}$ EMPLATE or pattern matching is a basic and fundamental image operation. In its simple form a given pattern is sought in an image, typically by scanning the image and evaluating a similarity measure between the pattern and every image window. Fast and reliable pattern matching is a basic building block in a vast range of applications, such as: image denoising, image re-targeting and summarization, image editing, super-resolution, object tracking, object recognition, and more (e.g. [6], [28], [18], [3]).

In most cases, however, the input image is acquired in an uncontrolled environment, thus, the sought pattern may vary in tone-levels due to changes in illumination conditions, camera photometric parameters, viewing positions, different modalities, etc. [20]. Commonly, these changes can be modeled locally by a non-linear tone mapping - a functional mapping between the gray-levels of the sought pattern and those of the image pattern. In this paper we deal with pattern matching where gray-levels may be subject to some unknown, possibly non-linear, tone mapping.

When dealing with matching under tone-mapping, three classes of approaches have been considered: the first class attempts to determine local signatures within the pattern and image that are invariant to tone mapping. Examples of this approach include Gradient Signatures [15], Histogram of Gradients [11], SIFT [21] and others (see [22] for comparative study).

- Y. Hel-Or is with the Department of Computer Science, The Interdisciplinary Center, Herzliya, Israel.

- H. Hel-Or and E. David are with the Department of Computer Science, University if Haifa, Haifa, Israel.
These signatures are often encoded to be invariant to geometric transformations as well as photometric variations. However, the data contraction implemented by these methods inherently involve loss of information and, thus, weaken their discrimination power. Consequently, these techniques often require an additional verification phase. Another approach to matching under tone-mapping, involves transformation of the pattern and image into a canonical configuration. Examples of this approach include Histogram Equalization and the well known Normalized CrossCorrelation (NCC) [5]. These approaches are limited in that there is no known canonical configuration for non-linear mappings. Finally, brute force methods attempt to perform template matching by searching the entire transformation parameter space, resulting in highly time consuming methods. Many distance measures for pattern matching have been suggested in the literature and the interested reader is referred to [10], [5] for excellent reviews. The approach suggested in this paper involves a search in the tone-mapping parameter space, however this search is performed very efficiently in closed form.

By far, the most common distance measure used for template matching is the Euclidean distance. Assume the pattern $\mathbf{p}$ and the candidate window $\mathbf{w}$ are both vectors in $\mathcal{R}^{m}$, (e.g. by raster scanning the pixels). The Euclidean distance between $\mathbf{p}$ and $\mathbf{w}$ is denoted: $d_{E}(\mathbf{p}, \mathbf{q})=\|\mathbf{p}-\mathbf{w}\|_{2}$. Searching for the minimal $d_{E}$ value in the image can be applied very fast using efficient convolution schemes [13]. Nevertheless, although very common, the Euclidean distance assumes no tone mapping has been applied thus it is inadequate when the image undergoes tone deformations.

To overcome tone mapping effects in images, the normalized cross correlation (NCC) distance is often 
used [5]. Consider the pattern $\mathbf{p}$ and the candidate window $\mathbf{w}$ as random variables with samples $p_{i}$ and $w_{i}, i=1 . . m$, respectively. The NCC is then defined as:

$$
\rho(\mathbf{p}, \mathbf{w})=E\left[\left(\frac{\mathbf{p}-E[\mathbf{p}]}{\sqrt{\operatorname{var}(\mathbf{p})}}\right)\left(\frac{\mathbf{w}-E[\mathbf{w}]}{\sqrt{\operatorname{var}(\mathbf{w})}}\right)\right]
$$

where for any vectors $\mathbf{x} \in \mathcal{R}^{m}, E[\mathbf{x}]$ and $\operatorname{var}(\mathbf{x})$ denote the empirical mean and variance. Due to the substraction of the mean and normalization by the s.t.d. in both $\mathbf{p}$ and $\mathbf{w}$, the NCC distance is invariant to linear tone mappings. The NCC distance can be applied very efficiently requiring little more than a single convolution on the input image [19]. However, such a distance will fail to detect patterns in cases where non-linear tone mappings have been applied ${ }^{1}$.

In many cases, the tone mapping is non-linear but still maintains monotonicity, namely, the order of tone-levels is preserved under the mapping. This scenario is common between images acquired using different cameras whose internal photometric parameters differ (tone correction, sensor spectral sensitivity, white balancing, etc). Image features that are based on ordinal values rather than the tone-levels themselves can account for monotonic mappings [32], [1]. Examples of such features, include Local Binary Pattern (LBP) [23], and Binary Robust Independent Elementary Features (BRIEF) [8]. Such representations are invariant to monotonic tone mapping and thus can be used to detect patterns in such cases. These approaches are fast to apply but are very sensitive to noise. Furthermore, these measures fail under nonmonotonic mappings.

Finally, when non-linear mapping is considered, Mutual Information (MI) is commonly used, initially proposed for image registration [30]. MI measures the statistical dependency between two variables. Clearly, the statistical dependency is strong when gray-levels of one image result from a functional mapping of the gray-levels of the other image. Thus, MI can account for non-linear mappings (both monotonic and nonmonotonic). In the context of pattern matching, MI measures the loss of entropy in the pattern $\mathbf{p}$ given a candidate window $\mathbf{w}$ :

$M I(\mathbf{p}, \mathbf{w})=H(\mathbf{w})-H(\mathbf{w} \mid \mathbf{p})=H(\mathbf{w})+H(\mathbf{p})-H(\mathbf{w}, \mathbf{p})$

where $H$ is the differential entropy.

Although MI is an effective similarity measure that can account for non-linear mappings, it is hindered by computational issues. First, it is computationally expensive as it requires the construction of the joint distribution (pattern vs. window) for each window to be matched. Although fast methods for evaluating histograms on running windows have been suggested [24], [31], fast methods for calculating local joint histograms are yet a challenge. Additionally, entropy as

1. NCC often performs well even under monotonic non-linear mappings as these can be assumed to be locally linear. well as MI is very sensitive to the size of histogram bins used to estimate the joint density, especially when sparse samples are given (small pattern size). Using kernel density estimation methods [29] rather than discrete histograms is, again, computationally expensive when dealing with joint probability, not to mention its sensitivity to the kernel width.

In this paper we propose a very fast pattern matching scheme termed Matching by Tone Mapping (MTM) which is invariant to non-linear tone mappings. The derivation of MTM is motivated by two complementary perspectives. One perspective considers the MTM as a regression problem where a non-linear tone mapping is sought to optimally fit the pattern to the candidate window. Thus, from its definition the MTM is invariant to non-linear tone mappings. The second perspective expresses MTM as a statistical measure which is shown to coincide with the correlation ratio [14]. The correlation ratio is a statistical measure that compares the dispersion of a given random variable with the dispersion of its conditional distribution. The correlation ratio was proposed by Roche et. al. [25], [26] as a distortion measure for multi-modal image registration. In this paper we show how this measure can be adapted to pattern matching, and suggest a very fast computational scheme requiring computational time similar to the fast implementation of NCC. Additionally, the regression perspective allows us to extend and modify the MTM beyond the correlation ratio to be more robust and appropriate for cases of small patches.

The MTM measure can be viewed as a generalization of the NCC for non-linear mappings. In fact, MTM reduces to NCC when mappings are restricted to be linear. We empirically show that the MTM is highly discriminative and robust to noise with comparable performance capability to that of the well performing Mutual Information. Thus, the MTM allows a pattern matching scheme on par with NCC in terms of computation time but with performance capability comparable to that of the Mutual Information scheme.

\section{Matching by Tone Mapping}

In the proposed pattern matching scheme, we wish to evaluate the minimum distance between a pattern and a candidate window under all possible tone mappings. Since tone mapping is not necessarily a bijective mapping, two alternatives may be considered: i) tone mapping applied to the pattern, transforming it to be as similar as possible to the candidate window, and ii) tone mapping applied to the window, transforming it to be as similar as possible to the pattern. For each case we find the minimum normed distance over all possible tone mappings.

Let $\mathbf{p} \in \mathcal{R}^{m}$ be a pattern and $\mathbf{w} \in \mathcal{R}^{m}$ a candidate window to be compared against. Denote by $\mathcal{M}: \mathcal{R} \rightarrow \mathcal{R}$ a tone mapping function. Thus, $\mathcal{M}(\mathbf{p})$ 
represents the tone mapping applied independently to each entry in $\mathbf{p}$. For the case of tone mapping applied to the pattern, the MTM distance is defined as follows:

$$
D(\mathbf{p}, \mathbf{w})=\min _{\mathcal{M}}\left\{\frac{\|\mathcal{M}(\mathbf{p})-\mathbf{w}\|^{2}}{m \operatorname{var}(\mathbf{w})}\right\}
$$

Similarly, if the mapping is applied to the window rather than the pattern, we define:

$$
D(\mathbf{w}, \mathbf{p})=\min _{\mathcal{M}}\left\{\frac{\|\mathcal{M}(\mathbf{w})-\mathbf{p}\|^{2}}{m \operatorname{var}(\mathbf{p})}\right\}
$$

The numerator in both cases is simply the norm distance after compensating for the tone mapping. The denominator is a normalization factor enforcing the distance to be scale invariant. Thus $D(\mathbf{p}, \mathbf{w})=$ $D(\mathbf{p}, \alpha \mathbf{w})$ for any scalar $\alpha$. Additionally, it penalizes incorrect matching of $\mathbf{p}$ to smooth windows when the constant mapping $\mathcal{M}(\mathbf{p})=c$ can be used. Due to the tone mapping compensation, the MTM measure reflects the inherent structural similarity between the pattern $\mathbf{p}$ and the window $\mathbf{w}$.

Searching for the pattern in the entire input image requires calculating the optimal tone mapping for each possible window in the image. Although seemingly a computationally expensive process, we show in the following sections that in fact this distance can be calculated very efficiently requiring an order of a single convolution with the input image!

\subsection{The Slice Transform (SLT)}

The Slice Transform (SLT) was first introduced in [17] in the context of Image Denoising. In this paper we exploit the SLT to represent a mapping function using a linear sum of basis functions. We first introduce a simplified version of the transform: the Piecewise Constant (PWC) case. Consider an image segment represented as a column vector $\mathbf{x}=\left[x_{1}, \cdots, x_{m}\right]^{T}$ with values in the half open interval $[a, b)$. The interval is divided into $k$ bins with boundary values $q_{1} \cdots q_{k+1}$ such that:

$$
a=q_{1}<q_{2}<\ldots<q_{k+1}=b
$$

Any value $v \in[a, b)$ is naturally associated with a single bin $\pi(v) \in\{1 \cdots k\}$ :

$$
\pi(v)=i \quad \text { if } \quad v \in\left[q_{i}, q_{i+1}\right)
$$

Given the bins defined by $\left\{q_{i}\right\}$, the vector $\mathbf{x}$ can be decomposed into a collection of binary slices: Slice $\mathbf{x}^{i}=\left[x_{1}^{i}, \cdots, x_{m}^{i}\right]$ is an indicator function over the vector $\mathbf{x}$ representing the entries of $\mathbf{x}$ associated with the $i$-th bin.

$$
x_{j}^{i}= \begin{cases}1 & \text { if } \pi\left(x_{j}\right)=i \\ 0 & \text { otherwise }\end{cases}
$$

The vector $\mathbf{x}$ can then be approximated as a linear combination of slice images:

$$
\mathbf{x} \approx \sum_{i=1}^{k} \alpha_{i} \mathbf{x}^{i}
$$

$$
\square \square S(x)=\begin{array}{|l|l|l|}
x^{1} & x^{2} & x^{3} \\
\hline & 0 & 0 \\
\hline 0 & 1 & 0 \\
\hline 0 & 0 & 1 \\
\hline 1 & 0 & 0 \\
\hline 0 & 0 & 1 \\
\hline
\end{array}
$$

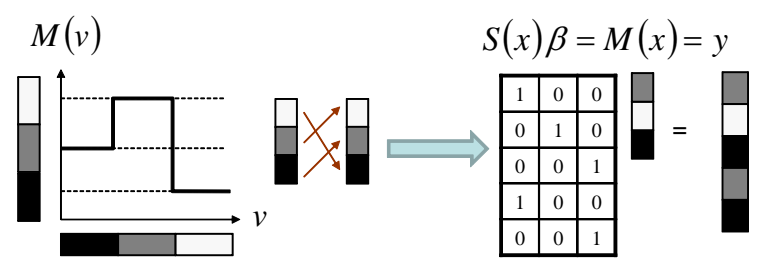

Fig. 1. Top: the SLT matrix for a 5-pixel vector having 3 gray values. Bottom: a piecewise constant mapping and its representation using the SLT matrix.

where the weights $\left\{\alpha_{i}\right\}_{i=1}^{k}$ are the values assigned to each bin (e.g $\alpha_{i}=q_{i}$ or $\left.\alpha_{i}=\left(q_{i}+q_{i+1}\right) / 2\right)$. The approximation is in fact a quantization of the values of $\mathbf{x}$ into the bins represented by $\left\{\alpha_{i}\right\}$. The greater the number of bins the better the approximation of the original image. In particular, if $\mathbf{x}$ values are discrete and $\forall j x_{j} \in\left\{q_{i}\right\}_{i=1}^{k+1}$ then $\mathbf{x}=\sum_{i=1}^{k} \alpha_{i} \mathbf{x}^{i}$.

Collecting the slices $\mathrm{x}^{i}$ in columns, we define the SLT matrix of $\mathbf{x}$ :

$$
S(\mathbf{x})=\left[\mathbf{x}^{1}, \mathbf{x}^{2}, \cdots, \mathbf{x}^{k}\right]
$$

Then Equation 4 can be rewritten in matrix form:

$$
\mathbf{x} \approx S(\mathbf{x}) \alpha
$$

where we define $\alpha=\left[\alpha_{1}, \alpha_{2}, \cdots, \alpha_{k}\right]^{T}$. Note, that since the slices are mutually disjoint, the columns of $S(\mathbf{x})$ are mutually orthogonal, satisfying:

$$
\mathbf{x}^{i} \cdot \mathbf{x}^{j}=\left|\mathbf{x}^{i}\right| \delta_{i, j}
$$

where ${ }^{\prime \prime}$. is the vectorial inner product, $|\mathbf{x}|$ denotes the cardinality of $\mathbf{x}$ and $\delta_{i, j}$ is the Kronecker's delta. The SLT matrix enables the representation of any piecewise constant mapping of $\mathbf{x}$; Substituting the vector $\alpha$ in Equation 6 with a different vector $\beta$, we obtain

$$
\mathbf{y}=S(\mathbf{x}) \beta
$$

Image $\mathbf{y}$ is a piecewise constant tone mapping of $\mathbf{x}$ s.t. all pixels of $\mathbf{x}$ with values in the $j$-th bin are mapped to $\beta_{j}$. Thus, the columns of $S(\mathbf{x})$ form an orthogonal basis spanning the space of all images that can be produced by applying a piecewise constant tone mapping on $\mathbf{x}$. Figure 1 illustrates an SLT matrix (top) and a piecewise mapping of a 5-pixel signal with 3 gray-level values (bottom). Figure 2 shows an example of linearly combining image slices to form the original (quantized) image (top row) and to form a tone mapped version (bottom row).

In the context of this paper, we use the SLT for tone mapping approximation. A mapping applied to 


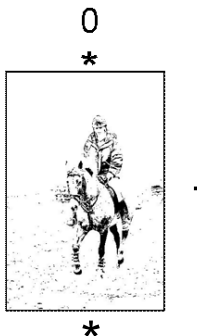

15

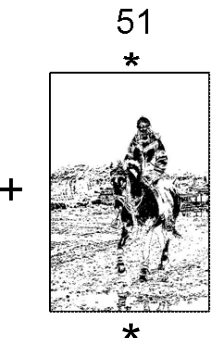

162

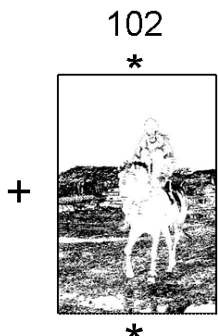

197

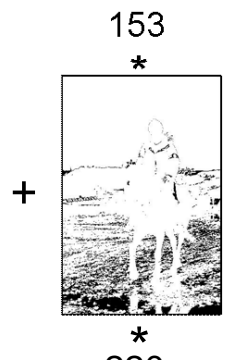

220

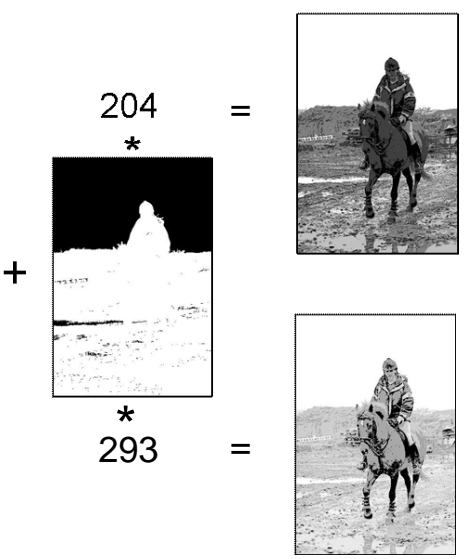

Fig. 2. Linear Combination of image slices. The SLT transform was applied to an image using 5 bins defined by $\alpha=[0,51,102,153,204,256]$. Using these $\alpha$ values as weights in the linear combination (top) reconstructs the original image. Using weights other than $\alpha$ (bottom) produces a tone mapped version of the original image. Slice images are shown inverted ( $1=$ black, $0=$ white).

pattern $\mathbf{p}$ is approximated by a piecewise constant mapping:

$$
\mathcal{M}(\mathbf{p}) \approx S(\mathbf{p}) \beta
$$

Consequently, the distance measures as defined in Equations 1-2 can be rewritten using the SLT:

$$
D(\mathbf{p}, \mathbf{w})=\min _{\beta} \frac{\|S(\mathbf{p}) \beta-\mathbf{w}\|^{2}}{m \operatorname{var}(\mathbf{w})}
$$

and similarly

$$
D(\mathbf{w}, \mathbf{p})=\min _{\beta} \frac{\|S(\mathbf{w}) \beta-\mathbf{p}\|^{2}}{m \operatorname{var}(\mathbf{p})}
$$

where $S(\mathbf{p})$ and $S(\mathbf{w})$ are the SLT matrices as defined in Equation 5. In the following sections we show that solving for $D$ for each image window can be applied very efficiently. In fact, computing $D$ over the entire image requires on the order of a single image convolution.

\subsection{MTM Distance Measure using SLT}

The SLT scheme allows a closed form solution for the minimizations defined in Equations 1 and 2. To introduce the matching process, we first consider the pattern-to-window case where a pattern $\mathbf{p}$ is to be matched against a candidate window $\mathbf{w}$. Thus, the distance measure used is that given in Equation 9. To simplify notation, we henceforth denote the SLT matrix $S(\mathbf{p})$ as $S$. The solution for $\beta$ that minimizes Equation 9 is given by:

$$
\hat{\beta}=\arg \min _{\beta}\|S \beta-\mathbf{w}\|^{2}=S^{\dagger} \mathbf{w}
$$

where $S^{\dagger}=\left(S^{T} S\right)^{-1} S^{T}$ is the Moore-Penrose pseudoinverse. Substituting into Equation 9 we obtain:

$$
D(\mathbf{p}, \mathbf{w})=\frac{\|S \hat{\beta}-\mathbf{w}\|^{2}}{m \operatorname{var}(\mathbf{w})}=\frac{\left\|S\left(S^{T} S\right)^{-1} S^{T} \mathbf{w}-\mathbf{w}\right\|^{2}}{m \operatorname{var}(\mathbf{w})}
$$

Due to the orthogonality of $S$, we have that $G=S^{T} S$ is a diagonal matrix with the histogram of $\mathbf{p}$ along its diagonal: $G(i, i)=\left|\mathbf{p}^{i}\right|$ where $\mathbf{p}^{i}$ is the pattern slice associated with the $i$-th bin as defined in Equation 3. Expanding the numerator it is easy to verify that:

$$
\left\|S\left(S^{T} S\right)^{-1} S^{T} \mathbf{w}-\mathbf{w}\right\|^{2}=\|\mathbf{w}\|^{2}-\left\|G^{-1 / 2} S^{T} \mathbf{w}\right\|^{2}
$$

Exploiting the diagonality of $G$ and using $S=$ $\left[\mathbf{p}^{1}, \mathbf{p}^{2}, \cdots, \mathbf{p}^{k}\right]$, the above expression can be rewritten using a sum of inner-products:

$$
\|\mathbf{w}\|^{2}-\left\|G^{-1 / 2} S^{T} \mathbf{w}\right\|^{2}=\|\mathbf{w}\|^{2}-\sum_{j} \frac{1}{\left|\mathbf{p}^{j}\right|}\left(\mathbf{p}^{j} \cdot \mathbf{w}\right)^{2}
$$

As a result, the overall MTM distance $D(\mathbf{p}, \mathbf{w})$ reads:

$$
D(\mathbf{p}, \mathbf{w})=\frac{1}{m \operatorname{var}(\mathbf{w})}\left[\|\mathbf{w}\|^{2}-\sum_{j} \frac{1}{\left|\mathbf{p}^{j}\right|}\left(\mathbf{p}^{j} \cdot \mathbf{w}\right)^{2}\right]
$$

In a similar manner, when matching is applied by mapping $\mathbf{w}$ towards $\mathbf{p}$ (window-to-pattern), we use Equation 10 and exchange the role of $\mathbf{w}$ and $\mathbf{p}$ to obtain a symmetric expression:

$$
D(\mathbf{w}, \mathbf{p})=\frac{1}{m \operatorname{var}(\mathbf{p})}\left[\|\mathbf{p}\|^{2}-\sum_{j} \frac{1}{\mid \mathbf{w}^{j \mid}}\left(\mathbf{w}^{j} \cdot \mathbf{p}\right)^{2}\right]
$$

\subsection{Calculating MTM Distance Over an Image}

Equations 11 and 12 provide a method for computing the structural difference between $\mathbf{p}$ and $\mathbf{w}$ using two complementary distances. For pattern matching, this computation must be performed on each candidate window of a given input image. Naively applying the above expressions to each image window is highly time consuming and impractical. In the following we show that, in fact, computing $D(\mathbf{p}, \mathbf{w})$ or $D(\mathbf{w}, \mathbf{p})$ over an entire image can be calculated very efficiently. We first describe the pattern-to-window mapping case, and then detail the window-to-pattern case. 


\section{P2W - Mapping pattern to window}

Let $\mathbf{F}$ be a 2D image with $n$ pixels in which pattern $\mathbf{p}$ is sought. Denote by $\mathbf{w}_{r}$ the $r$-th window of $\mathbf{F}$. Consider the pattern-to-window (P2W) scheme where the distance given in Equation 11 is used. For each window $\mathbf{w}_{r} \in F$ two terms must be calculated, namely the numerator $d_{1}$ and the denominator $d_{2}$ :

$d_{1}(r)=\left\|\mathbf{w}_{r}\right\|^{2}-\sum_{j} \frac{1}{\left|\mathbf{p}^{j}\right|}\left(\mathbf{p}^{j} \cdot \mathbf{w}_{r}\right)^{2}, d_{2}(r)=m \operatorname{var}\left(\mathbf{w}_{r}\right)$

Since computing the inner-product over all windows can be performed using image convolution, the terms above can be calculated efficiently. We use $\operatorname{var}\left(\mathbf{w}_{r}\right)=$ $E\left[\mathbf{w}_{r}^{2}\right]-E^{2}\left[\mathbf{w}_{r}\right]$ to efficiently calculate the denominator. Algorithm 1 gives the pseudo-code for calculating the P2W MTM distance between pattern $\mathbf{p}$ and each window in $F$ (code can be found in [16]). In the pseudo-code ' $*$ ' denotes image convolution, $\odot$ and $\oslash$ denote elementwise multiplication and division, respectively. Upper-case letters denote arrays of size similar to the image $F$, and lower-case letters denote scalar variables. Vectors and filter kernels are denoted by bold lower-case letters. $\mathbf{1}$ is an m-vector of 1's (box filter). Since correlations rather than convolutions are required, flipped kernels are used when needed.

Prior to the loop, two convolutions with a box filter are calculated, each of which can be applied efficiently (with a separable 1D box filter) requiring a total of 4 additions per pixel. Within the loop there are $k$ convolutions with the pattern slices $\left\{\mathbf{p}^{j}\right\}_{j=1}^{k}$. Since each slice $\mathbf{p}^{j}$ is sparse, convolving it with an image requires only $\left|\mathbf{p}^{j}\right|$ additions per pixel using a sparse convolution scheme [33]. Additionally, since all pattern slices are mutually disjoint the total number of additions per pixel sum to $m$. All other operations sum to $O(k)$ operations per pixel, thus, the algorithm requires a total of $O(m n+k n)$ operations which is comparable in complexity to a single convolution! Memory requirement is also economized: distance

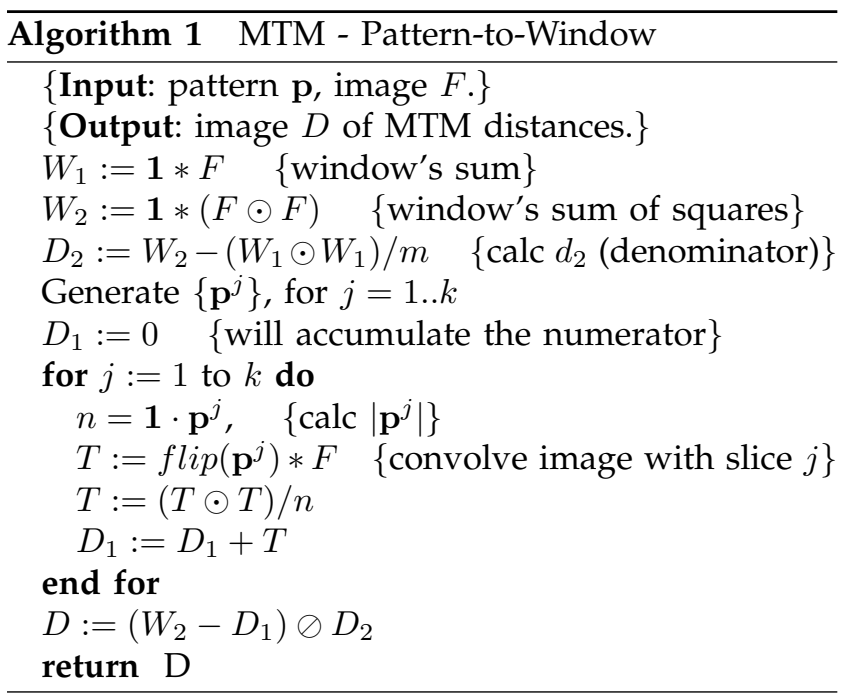

value for each image window is accumulated in place, requiring memory on the order of image size.

\section{W2P - Mapping window to pattern}

Consider now the window-to-pattern (W2P) scheme using the distance given in Equation 12. For each window $\mathbf{w}_{r} \in F$, the expressions to be calculated are:

$d_{1}(r)=\|\mathbf{p}\|^{2}-\sum_{j} \frac{1}{\left|\mathbf{w}_{r}^{j}\right|}\left(\mathbf{w}_{r}^{j} \cdot \mathbf{p}\right)^{2} \quad$ and $\quad d_{2}=m \operatorname{var}(\mathbf{p})$

$d_{2}$ and the first term of $d_{1}$ are constant for all windows and are calculated only once. The second term in $d_{1}$ differs for each window. Algorithm 2 gives the pseudo-code for calculating the W2P distance over the entire image. In this algorithm $F^{j}$ denotes the $j$ th image slice, i.e. $F^{j}(x, y)=1$ iff $\pi(F(x, y))=j$. Since each $F^{j}$ is a sparse image, convolution can be applied efficiently in this case as well. Note that $\left\{F^{j}\right\}$ are mutually disjoint, thus the operations required for the $k$ sparse convolutions sum to $O(m n)$ operations. As in the P2W case, the entire algorithm requires $O(m n+k n)$ operations, which is on the order of a single image convolution. Memory requirement is also economical and is on the order of the image size.

\section{Statistical Properties}

In this section we give statistical justification for the proposed distance. Throughout this section we discuss the pattern-to-window case where $D(\mathbf{p}, \mathbf{w})$ distance is used. All observations and claims are applicable symmetrically in the window-to-pattern case (using $D(\mathbf{w}, \mathbf{p})$ ).

Recall that $D(\mathbf{p}, \mathbf{w})$ is composed of two terms:

$$
D(\mathbf{p}, \mathbf{w})=\frac{d_{1}}{d_{2}}=\frac{\|\mathbf{w}\|^{2}-\sum_{j} \frac{1}{\left|\mathbf{p}^{j}\right|}\left(\mathbf{p}^{j} \cdot \mathbf{w}\right)^{2}}{m \cdot \operatorname{var}(\mathbf{w})}
$$

Theorem 1 states that $D(\mathbf{p}, \mathbf{w})$ measures the ratio between the conditional variance of $(\mathbf{w} \mid \mathbf{p})$ and the variance of $\mathbf{w}$.

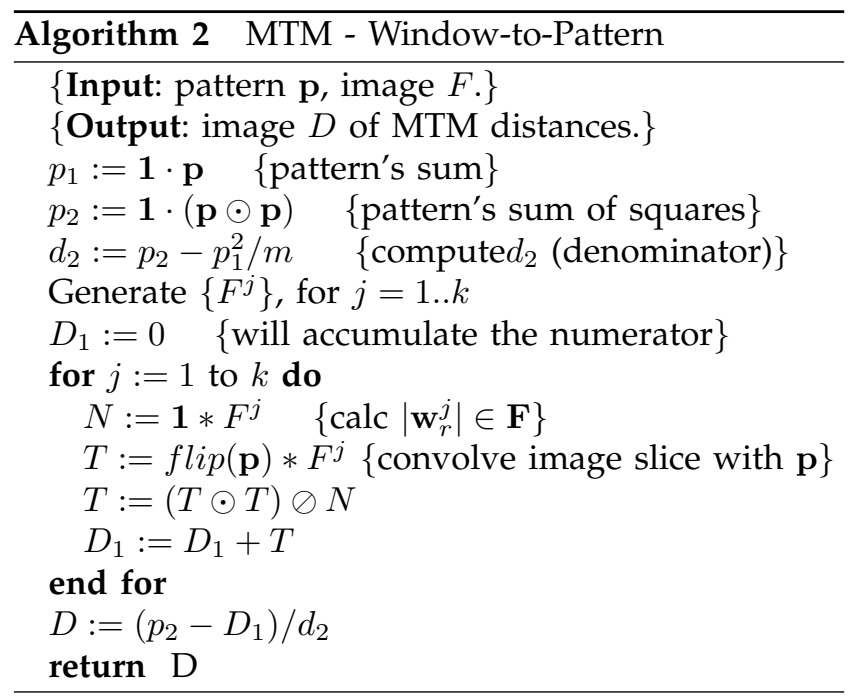




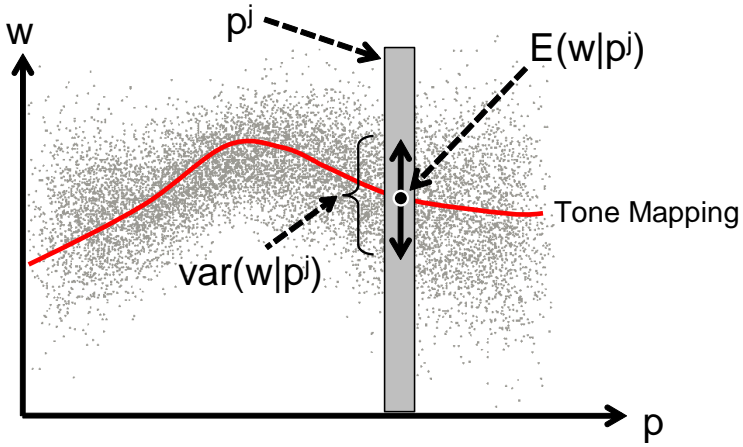

Fig. 3. Conditional expectation $E\left[\operatorname{var}\left(\mathbf{w} \mid \mathbf{p}^{j}\right)\right]$.

Theorem 1.

$$
D(\mathbf{p}, \mathbf{w})=\frac{E[\operatorname{var}(\mathbf{w} \mid \mathbf{p})]}{\operatorname{var}(\mathbf{w})}
$$

where $E[\cdot]$ is taken over all sample values $p_{i}$ of $\mathbf{p}$.

Proof 1 Let $\mathbf{p}^{j}$ be the $j$-th slice of $\mathbf{p}$. All pattern slices are mutually exclusive, thus $\sum_{j} \mathbf{p}^{j}=\mathbf{1}$. Consequently, $d_{1}$ can be rewritten as:

$$
\begin{aligned}
d_{1} & =\sum_{j} \mathbf{p}^{j} \cdot(\mathbf{w} \odot \mathbf{w})-\sum_{j} \frac{\left(\mathbf{p}^{j} \cdot \mathbf{w}\right)^{2}}{\left|\mathbf{p}^{j}\right|} \\
& =\sum_{j}\left|\mathbf{p}^{j}\right| \frac{\mathbf{p}^{j} \cdot(\mathbf{w} \odot \mathbf{w})}{\left|\mathbf{p}^{j}\right|}-\sum_{j}\left|\mathbf{p}^{j}\right|\left(\frac{\mathbf{p}^{j} \cdot \mathbf{w}}{\left|\mathbf{p}^{j}\right|}\right)^{2} \\
& =\sum_{j}\left|\mathbf{p}^{j}\right|\left(\frac{\mathbf{p}^{j} \cdot(\mathbf{w} \odot \mathbf{w})}{\left|\mathbf{p}^{j}\right|}-\left(\frac{\mathbf{p}^{j} \cdot \mathbf{w}}{\left|\mathbf{p}^{j}\right|}\right)^{2}\right)
\end{aligned}
$$

Considering $\mathbf{p}$ and $\mathbf{w}$ as random variables with $m$ samples $p_{i}$ and $w_{i}, i=1 . . m$, respectively we have:

$$
\frac{\mathbf{p}^{j} \cdot \mathbf{w}}{\left|\mathbf{p}^{j}\right|}=E\left[\mathbf{w} \mid \mathbf{p}^{j}\right]
$$

and

$$
\begin{aligned}
d_{1} & =\sum_{j}\left|\mathbf{p}^{j}\right|\left(E\left[\mathbf{w} \odot \mathbf{w} \mid \mathbf{p}^{j}\right]-E^{2}\left[\mathbf{w} \mid \mathbf{p}^{j}\right]\right) \\
& =\sum_{j}\left|\mathbf{p}^{j}\right| \operatorname{var}\left(\mathbf{w} \mid \mathbf{p}^{j}\right)=m E[\operatorname{var}(\mathbf{w} \mid \mathbf{p})]
\end{aligned}
$$

where the expectation in the last equation is taken over all $\left\{\mathbf{p}^{j}\right\}$. From Equation 13 it follows that

$$
D(\mathbf{p}, \mathbf{w})=\frac{d_{1}}{d_{2}}=\frac{E[\operatorname{var}(\mathbf{w} \mid \mathbf{p})]}{\operatorname{var}(\mathbf{w})}
$$

The interpretation of this Theorem is given in Figure 3 where a scatter diagram of a specific pair $\mathbf{p}$ and $\mathbf{w}$ is shown. The horizontal axis indicates pattern values and the vertical axis indicates corresponding window values. Each pair of values is represented as a point in the scatter diagram. The empirical mean $E\left[\mathbf{w} \mid \mathbf{p}^{j}\right]$ for the $j^{t h}$ bin is drawn as a full circle, and the conditional variance $\operatorname{var}\left(\mathbf{w} \mid \mathbf{p}^{j}\right)$ is illustrated as a double headed arrow. Note, that in terms of MTM matching, $E\left[\mathbf{w} \mid \mathbf{p}^{j}\right]$ is the estimated tone map value for the tones in $\mathbf{p}$ associated with the $j^{\text {th }}$ bin. The expectation value of $\operatorname{var}\left(\mathbf{w} \mid \mathbf{p}^{j}\right)$ over all bins $\mathbf{p}^{j}, j=1 . . k$ is $E[\operatorname{var}(\mathbf{w} \mid \mathbf{p})]$. Intuitively, this evaluates the spread of the data around the estimated tone mapping. Thus, Theorem 1 implies that when seeking a good match for $\mathbf{p}$ over the entire image, a candidate window $\mathbf{w}$ is sought whose values are tight around the tone mapped pattern and concurrently is of high variance (thus penalizing uniform and smooth windows). Note, however, that rather than minimizing $D(\mathbf{p}, \mathbf{w})$ one can equivalently maximize:

$$
\tilde{D}(\mathbf{p}, \mathbf{w})=1-D(\mathbf{p}, \mathbf{w})=\frac{\operatorname{var}(\mathbf{w})-E[\operatorname{var}(\mathbf{w} \mid \mathbf{p})]}{\operatorname{var}(\mathbf{w})}
$$

which is the normalized reduction in the variance of $\mathbf{w}$ when $\mathbf{p}$ is given. This relation bears a strong similarity to the mutual-information measure. In both cases the goal is to maximize the reduction in the uncertainty of $\mathbf{w}$ given $\mathbf{p}$. However, while the MI scheme uses entropy as the uncertainty measure, the MTM uses variance as the uncertainty measure. Using variance rather than entropy enables the MTM scheme to be applied very fast on large images. Additionally, while the MI measure is very sensitive to the size of the bins (or the width of the kernel, if kernel estimation is used), the performance of the variance based MTM measure is not much affected by varying the number of bins. Further discussion on MTM vs MI can be found in Section 5.

As stated above, the empirical mean $E\left[\mathbf{w} \mid \mathbf{p}^{j}\right]$ for the $j^{\text {th }}$ bin (full circle in Figure 3 ) is the estimated tone-mapping for the values in $\mathbf{p}^{j}$. The collection $\left\{E\left[\mathbf{w} \mid \mathbf{p}^{j}\right]\right\}$ for all $j=1 . . k$, forms the estimated optimal tone mapping that maps $\mathbf{p}$ to $\mathbf{w}$ (solid curve in Figure 3). The variance of the collection $\left\{E\left[\mathbf{w} \mid \mathbf{p}^{j}\right]\right\}$ is closely related to the MTM distance $D(\mathbf{p}, \mathbf{w})$. We state this relation in the following theorem:

\section{Theorem 2.}

$$
\tilde{D}(\mathbf{p}, \mathbf{w})=1-D(\mathbf{p}, \mathbf{w})=\frac{\operatorname{var}(E[\mathbf{w} \mid \mathbf{p}])}{\operatorname{var}(\mathbf{w})}
$$

\section{Proof 2.}

The theorem is derived directly from the law of total variance [27] which states:

$$
\operatorname{var}(\mathbf{w})=E[\operatorname{var}(\mathbf{w} \mid \mathbf{p})]+\operatorname{var}(E[\mathbf{w} \mid \mathbf{p}])
$$

Therefore,

$\tilde{D}(\mathbf{p}, \mathbf{w})=\frac{\operatorname{var}(\mathbf{w})-E[\operatorname{var}(\mathbf{w} \mid \mathbf{p})]}{\operatorname{var}(\mathbf{w})}=\frac{\operatorname{var}(E[\mathbf{w} \mid \mathbf{p}])}{\operatorname{var}(\mathbf{w})}$

Note that the term $\tilde{D}(\mathbf{p}, \mathbf{w})$ is the Correlation Ratio statistical measure [14]. Roche et. al. suggested to use this measure for multi-modal image registration [25].

Theorem 2 implies that the mean of the conditional variance and the variance of the conditional mean are interchangeable, in the sense that, minimization over the first is the maximization over the latter:

$$
\arg \min _{\mathbf{w}} \frac{E[\operatorname{var}(\mathbf{w} \mid \mathbf{p})]}{\operatorname{var}(\mathbf{w})}=\arg \max _{\mathbf{w}} \frac{\operatorname{var}(E[\mathbf{w} \mid \mathbf{p}])}{\operatorname{var}(\mathbf{w})}
$$


Both measures are in the range $[0,1]$. When the optimal tone mapping is uniform, i.e. $\exists c$, s.t. $E\left(\mathbf{w} \mid \mathbf{p}^{j}\right)=c$ for $j=1 . . k$, then $\operatorname{var}(E[\mathbf{w} \mid \mathbf{p}])=0, E[\operatorname{var}(\mathbf{w} \mid \mathbf{p})]=\operatorname{var}(\mathbf{w})$ and $\tilde{D}(\mathbf{p}, \mathbf{w})=0$ while $D(\mathbf{p}, \mathbf{w})=1$. Thus, although the $\mathbf{w}$ values are well predictable from $\mathbf{p}$, the MTM distance is still large since the predictability is only due to the low dispersion of $\mathbf{w}$. This property is imperative for pattern matching, since the $\mathrm{w}$ values located in smooth image regions (such as sky or nontextured surfaces) are predictable from $\mathbf{p}$, but this is not the desired matching solution. Note, that this is also the reason that the mutual information was preferred over the conditional entropy in [30].

Additionally, it can be shown using the law of total variance [27] that

$$
\arg \max _{\mathbf{w}} \frac{\operatorname{var}(E[\mathbf{w} \mid \mathbf{p}])}{\operatorname{var}(\mathbf{w})}=\arg \max _{\mathbf{w}} \frac{\operatorname{var}(E[\mathbf{w} \mid \mathbf{p}])}{E[\operatorname{var}(\mathbf{w} \mid \mathbf{p})]}
$$

which yields that MTM is related to the Fisher Linear Discriminant [12], in which the goal is to maximize inter-class variance (numerator) while minimizing intra-class variance (denominator), where, in our case, each bin takes the role of a class.

Finally, we show that the MTM scheme is a generalization of the NCC distance measure. In particular, when tone mappings are restricted to be linear functions, the NCC and MTM distances coincide [25]:

Theorem 3. Assume tone mappings are restricted to be linear, i.e. $\mathcal{M}(\mathbf{p})=a \mathbf{p}+b$, where $a, b$ are scalar parameters. Denoting by $\rho(\mathbf{p}, \mathbf{w})$, the normalized cross correlation distance as defined in Equation 1, we have:

$$
\tilde{D}(\mathbf{p}, \mathbf{w})=\rho^{2}(\mathbf{p}, \mathbf{w})
$$

Proof 3. Considering the original definition of MTM (Equation 1) under the restriction to linear mappings: $\mathcal{M}(\mathbf{p})=a \mathbf{p}+b$, we seek parameters $a, b$ satisfying:

$$
\min _{a, b}\|a \mathbf{p}+b-\mathbf{w}\|^{2}
$$

It has been shown (e.g. [27] Ch. 7) that minimizing the above term gives:

$$
\begin{aligned}
a & =\frac{\operatorname{cov}(\mathbf{p}, \mathbf{w})}{\operatorname{var}(\mathbf{p})}=\rho(\mathbf{p}, \mathbf{w}) \sqrt{\frac{\operatorname{var}(\mathbf{w})}{\operatorname{var}(\mathbf{p})}} \\
b & =E[\mathbf{w}]-a E[\mathbf{p}]=E[\mathbf{w}]-\rho(\mathbf{p}, \mathbf{w}) E[\mathbf{p}] \sqrt{\frac{\operatorname{var}(\mathbf{w})}{\operatorname{var}(\mathbf{p})}}
\end{aligned}
$$

where $\rho(\mathbf{p}, \mathbf{w})=\frac{\operatorname{cov}(\mathbf{p}, \mathbf{w})}{\sqrt{\operatorname{var}(\mathbf{p}) \operatorname{Var}(\mathbf{w})}}$. Substituting $a$ and $b$ into Equation 1 we obtain [27]:

$$
\tilde{D}(\mathbf{p}, \mathbf{w})=1-D(\mathbf{p}, \mathbf{w})=\rho^{2}(\mathbf{p}, \mathbf{w})
$$

\section{Piecewise Linear MTM}

The benefits of using the piecewise constant (PWC) approximation for tone mappings as suggested in Section 2.1 are simplicity and computational efficiency.
This approximation allows flexible functional relationships between the pattern and the sought window. In some cases, however, this flexibility introduces a weakness as it generates over-fitting solutions for SLT bins having very few samples. Such scenarios occur mainly when small sized patterns are sought. Increasing the bin sizes does not solve this problem as it also increases the mapping representation error. To allow larger bin sizes without degrading the modeling precision we extend the SLT transform to implement a higher order regression model, namely, a Piecewise Linear (PWL) approximation. This model approximates the tone mapping as a piecewise linear function and enables aggregating more samples into each bin without compromising representation accuracy. Similar to the PWC-SLT, the PWL-SLT slices a given image into $k$ slices, but rather than being binary slices, the slices now contain real values.

Recall the SLT definition described in Section 2.1, we denote bin boundaries as a sequence $q_{1}, \cdots, q_{k+1}$ where $q_{1}<q_{2}<\cdots<q_{k+1}$. A value $x$ in the half open interval $\left[q_{1}, q_{k+1}\right)$ is associated with a bin $\pi(x)$

$$
\pi(x)=j \quad \text { if } x \in\left[q_{j}, q_{j+1}\right)
$$

We define $r(x)$ to be the relative position of $x$ in its bin:

$$
r(x)=\frac{x-q_{\pi(x)}}{q_{\pi(x)+1}-q_{\pi(x)}}
$$

Note, that $r(x) \in[0,1]$, where $r(x)=0$ if $x=q_{\pi(x)}$, and $r(x) \rightarrow 1$ when $x \rightarrow q_{\pi(x)+1}$. For every $x \in$ $\left[q_{1}, q_{k+1}\right)$ the following relation holds:

$$
x=(1-r(x)) \cdot q_{\pi(x)}+r(x) \cdot q_{\pi(x)+1}
$$

Defining a $k+1$ dimensional vector $\alpha$ as a vector composed of the bin boundaries:

$$
\alpha=\left[q_{1}, q_{2}, \cdots, q_{k+1}\right]
$$

Equation 15 can be rewritten in vectorial form:

$$
x=Q(x) \alpha
$$

where $Q(x)$ is a row vector:

$$
Q(x)=[0, \cdots, 0,1-r(x), r(x), 0, \cdots, 0]
$$

s.t. the values $1-r(x)$ and $r(x)$ are located in the $\pi(x)$ and $\pi(x)+1$ entries, respectively. We now define a matrix extension of Equation 16. Let $\mathbf{x} \in \mathcal{R}^{m}$ be a real valued vector whose elements satisfy $x_{i} \in\left[q_{1}, q_{k+1}\right)$. The piecewise linear slice transform (PWL-SLT) of $\mathbf{x}$ is defined as:

$$
\mathbf{x}=Q(\mathbf{x}) \alpha
$$

where $Q(\mathbf{x})$ is an $m \times(k+1)$ SLT matrix:

$$
[Q(\mathbf{x})](i, j)= \begin{cases}1-r\left(x_{i}\right) & \text { if } \pi\left(x_{i}\right)=j \\ r\left(x_{i}\right) & \text { if } \pi\left(x_{i}\right)=j-1 \\ 0 & \text { otherwise }\end{cases}
$$

Note that, in contrast with the PWC case, multiplying $Q(\mathbf{x})$ with the vector $\alpha$ does not quantize $\mathbf{x}$ but 
reproduces $x$ exactly (Equation 17), regardless of the number of bins. Substituting the boundary vector in the expression $Q(\mathbf{x}) \alpha$ with a different vector $\beta$ we obtain a piecewise linear tone mapping of $\mathrm{x}$ :

$$
\mathbf{y}=Q(\mathbf{x}) \beta
$$

This mapping implies that values in the interval $\left[\alpha_{i}, \alpha_{i+1}\right)$ are linearly mapped to the interval $\left[\beta_{i}, \beta_{i+1}\right)$. Note, that in contrast with the PWC-SLT matrix $S(\mathbf{x})$, the columns of matrix $Q(\mathbf{x})$ are not orthogonal. Thus we use a variant of the original image slice defined in Section 2.1:

We define $\tilde{\mathbf{x}}^{j}=\left[\tilde{x}_{1}^{j}, \cdots, \tilde{x}_{m}^{j}\right]$ as a real valued vector associated with the $j^{\text {th }}$ bin:

$$
\tilde{x}_{i}^{j}= \begin{cases}r\left(x_{i}\right) & \text { if } \pi\left(x_{i}\right)=j \\ 0 & \text { otherwise }\end{cases}
$$

The matrix $Q(\mathbf{x})$ can then be represented as a collection of column vectors (slices):

$$
Q(\mathbf{x})=\left[\overline{\mathbf{x}}^{1}, \overline{\mathbf{x}}^{2}, \cdots, \overline{\mathbf{x}}^{k+1}\right]
$$

where we define

$$
\overline{\mathbf{x}}^{j}=\mathbf{x}^{j}-\tilde{\mathbf{x}}^{j}+\tilde{\mathbf{x}}^{j-1}
$$

where $\tilde{\mathbf{x}}^{j}$ is defined above (Equation 20), and $\mathbf{x}^{j}$ is the originally defined slice vector (Equation 3 ). The end cases are set to be: $\mathbf{x}^{k+1}=\tilde{\mathbf{x}}^{k+1}=\tilde{\mathbf{x}}^{0}=\mathbf{0}$.

The PWL-SLT is used to compute the piecewise linear MTM (MTM-PWL) efficiently on the entire image. In this case, the minimum normed distance between a pattern and candidate window (Equations 1 and 2) is evaluated under all possible piecewise linear tone mappings. Since tone mapping is not necessarily bijective, two alternatives must again be considered: Pattern-toWindow (P2W) and Window-to-Pattern (W2P).

\subsection{P2W by Piecewise Linear Mapping}

The MTM distance is given by the minimization of Equation 1 where $\mathcal{M}(\mathbf{p})=Q(\mathbf{p}) \beta$. The optimal mapping is then given by:

$$
\hat{\beta}=\arg \min _{\beta}\|Q \beta-\mathbf{w}\|^{2}=\left(Q^{T} Q\right)^{-1} Q^{T} \mathbf{w}
$$

For simplicity we denote $Q(\mathbf{p})$ by $Q$. Substituting back into Equation 1, the MTM distance reads:

$$
D(\mathbf{p}, \mathbf{w})=\frac{\|Q \hat{\beta}-\mathbf{w}\|^{2}}{m \cdot \operatorname{var}(\mathbf{w})}=\frac{\left\|Q\left(Q^{T} Q\right)^{-1} Q^{T} \mathbf{w}-\mathbf{w}\right\|^{2}}{m \cdot \operatorname{var}(\mathbf{w})}
$$

Expanding the numerator, we obtain:

$d_{1}=\left\|Q\left(Q^{T} Q\right)^{-1} Q^{T} \mathbf{w}-\mathbf{w}\right\|^{2}=\|\mathbf{w}\|^{2}-\left(\mathbf{w}^{T} Q G^{-1} Q^{T} \mathbf{w}\right)$

where $G=Q^{T} Q$. Unlike the PWC case, the matrix $G$ in this case is not diagonal, thus calculating the right hand term in Equation 23 is more challenging than the PWC case. Fortunately, $G$ is a tridiagonal matrix, a property we exploit to expedite calculations. Note,

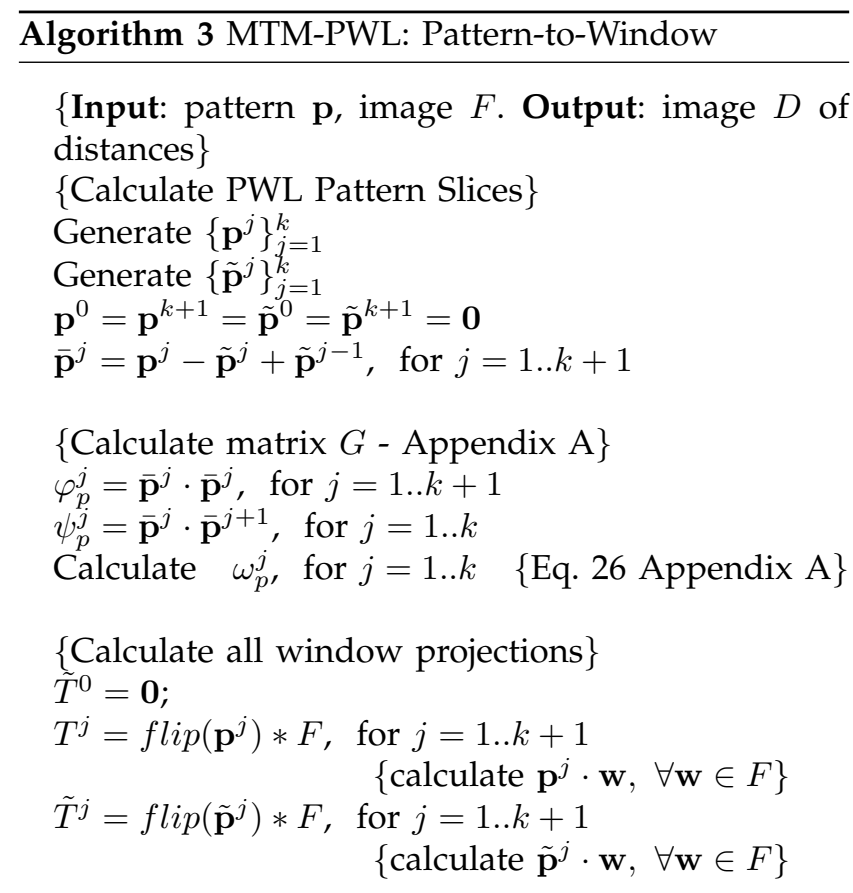

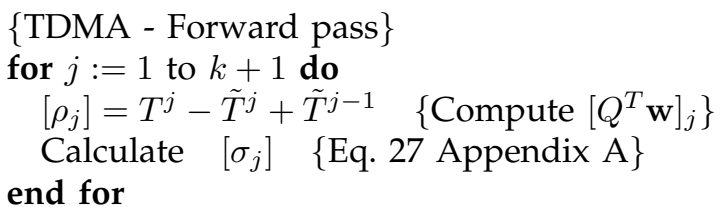

\{TDMA - Backward pass $\}$

for $j:=k+1$ to 1 do

Calculate $\left[\hat{\beta}_{j}\right] \quad$ \{Eq. 28 Appendix A\} end for

\{Calculate distances for all windows

$D_{1}=\sum_{j}\left[\hat{\beta}_{j}\right] \odot\left[\rho_{j}\right] \quad\left\{\right.$ calc $d_{1}$ (numerator) $\}$

$W_{1}:=1 * F \quad$ \{window sum

$W_{2}:=\mathbf{1} *(F \odot F) \quad$ \{window sum of squares

$D_{2}:=W_{2}-\left(W_{1} \odot W_{1}\right) / m \quad$ \{calc $d_{2}$ (denominator) $\}$

$D:=\left(W_{2}-D_{1}\right) \oslash D_{2}$

return $\mathrm{D}$

that in the P2W case, $G$ is a function of $\mathbf{p}$ and may be calculated only once for all candidate windows.

Recall that the columns $\overline{\mathbf{p}}^{j}$ of $Q$ are given by Equations 20 and 21. Thus calculating $Q^{T} \mathbf{w}$ in Equation 23 requires $2 k$ dot products $\left\{\mathbf{w} \cdot \mathbf{p}^{j}\right\}_{j=1}^{k}$ and $\left\{\mathbf{w} \cdot \tilde{\mathbf{p}}^{j}\right\}_{j=1}^{k}$ :

$$
\rho_{j}=\left[Q^{T} \mathbf{w}\right]_{j}=\mathbf{w} \cdot \overline{\mathbf{p}}^{j}=\mathbf{w} \cdot\left(\mathbf{p}^{j}-\tilde{\mathbf{p}}^{j}+\tilde{\mathbf{p}}^{j-1}\right)
$$

However, since the pattern slices are mutually exclusive, the $k$ dot-products with $\left\{\mathbf{p}^{j}\right\}$ as well as with $\left\{\tilde{\mathbf{p}}^{j}\right\}$ require only $O(m+k)$ operations, for each. Calculating the entire term $\mathbf{w}^{T} Q G^{-1} Q^{T} \mathbf{w}$ requires multiplication of $Q^{T} \mathbf{w}$ with $G^{-1}$. Since $G^{-1}$ is a $k \times k$ matrix, this would require an additional $k^{2}$ operations. However, since $G$ is tridiagonal we use the Tridiagonal Matrix Algorithm (TDMA) [9] as follows. Denote $\hat{\beta}=G^{-1} Q^{T} \mathbf{w}$, thus $G \hat{\beta}=Q^{T} \mathbf{w}$. Using TDMA, solving for $\hat{\beta}$ can be 

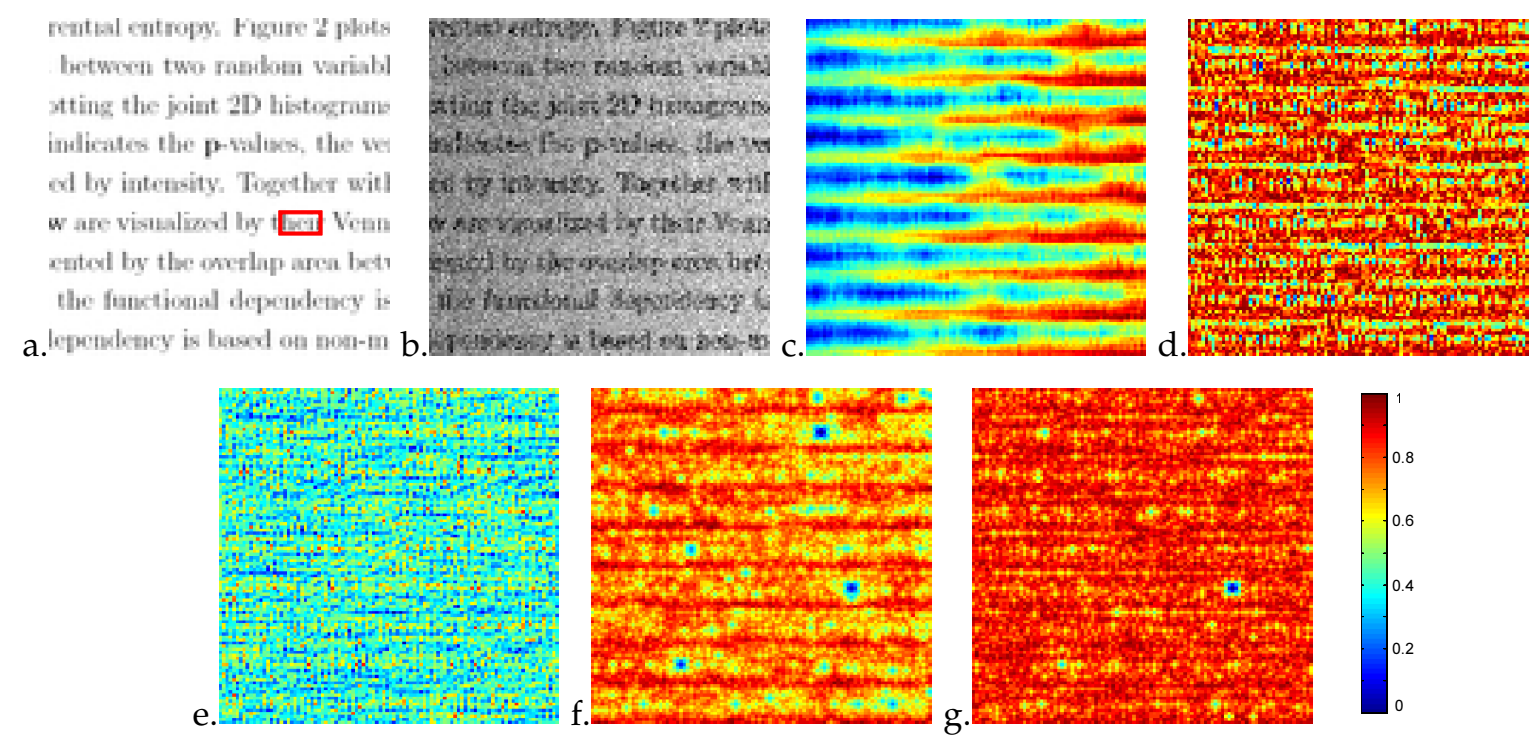

Fig. 4. Pattern marked in (a) is sought in tone mapped image with added noise (b). Distance maps display distance values between pattern and image windows for: (c) Euclidean (d) NCC (e) LBP (f) MI (g) MTM.

implemented in $O(m+k)$ operations using Gaussian elimination and backward substitution (Appendix A). Therefore, calculating the entire term $\mathbf{w}^{T} Q G^{-1} Q^{T} \mathbf{w}$ requires $O(2(m+k))$ operations. Algorithm 3 gives a pseudo-code for applying P2W pattern matching over an entire image F using PWL approximation. In the pseudo-code capital letters and bracketed variables (such as $\left[\rho_{j}\right]$ ) represent images of size equal to $F$. Assuming the image $\mathrm{F}$ is of $n$ pixels, the entire search requires $O(2(n m+n k))$ operations, which is equivalent to two image convolutions!

\subsection{W2P by Piecewise Linear Mapping}

Due to symmetry in roles of $\mathbf{p}$ and $\mathbf{w}$, they can be interchanged in Equations 22 and 23 obtaining:

$$
D(\mathbf{w}, \mathbf{p})=\frac{\|Q \hat{\beta}-\mathbf{p}\|^{2}}{m \cdot \operatorname{var}(\mathbf{p})}=\frac{\|\mathbf{p}\|^{2}-\mathbf{p}^{T} Q G^{-1} Q^{T} \mathbf{p}}{m \cdot \operatorname{var}(\mathbf{p})}
$$

where $Q=Q(\mathbf{w})$ and $G=Q^{T} Q$. The scalars $\|\mathbf{p}\|^{2}$ and $\operatorname{var}(\mathbf{p})$ are calculated once for all windows, however, the term $\mathbf{p}^{T} Q G^{-1} Q^{T} \mathbf{p}$ must now be calculated explicitly for each window in $F$. In this case, we denote

$$
\rho_{j}=\left[Q^{T} \mathbf{p}\right]_{j}=\mathbf{w}^{j} \cdot \overline{\mathbf{p}}
$$

where

$$
\overline{\mathbf{w}}^{j}=\mathbf{w}^{j}-\tilde{\mathbf{w}}^{j}+\tilde{\mathbf{w}}^{j+1}
$$

We again use the tridiagonal property of $G$ and the TDMA algorithm to produce the MTM distance for each window in image $F$ with $O(2(n k+n m))$ operations. The algorithm for calculating $D(\mathbf{w}, \mathbf{p}), \forall \mathbf{w} \in F$ is similar to Algorithm 3 with roles reversed. Thus the slice transform is applied to the image rather than the pattern and pattern projections are computed rather than window projections.

\section{Results on Simulated Data}

The suggested method was compared with four distance measures discussed in Section 1, namely, the Euclidean distance(EUC), Local Binary Pattern (LBP), Normalized Cross Correlation (NCC), and Mutual Information (MI). In this section we show the MTM approach successfully and efficiently detects the sought patterns, under extreme tone mappings and under heavy noise conditions, performing on par and at times better than the MI approach and significantly better than the other compared methods, while maintaining run times significantly lower than MI.

To illustrate performance, consider the image and the selected pattern in Figure 4a. Figure $4 \mathrm{~b}$ shows the original image after applying non-linear tone mapping, adding a global illumination gradient and adding white Gaussian noise. The selected pattern (size 10x20) was sought in the tone mapped image by evaluating the distance between the pattern and every window in the image using the five distance measures. Bin sizes (MI of size 20 and MTM-PWL of size 40) were chosen to provide best results. Figures $4 \mathrm{c}-\mathrm{g}$ show the resulting maps. It can be seen that the MTM distance clearly shows a sharp peak at the correct location overcoming both non-monotonic mapping and noise. The Euclidean and the LBP measures both strongly fail due to the non linearity of the mapping and due to the noise. The NCC, fails due to the non-linearity of the tone mapping. The MI shows confusion in the detection locations, this is mainly due to the relatively small pattern size which implies very sparse data in the MI bins (even when bin size increases to 40 gray values).

Pattern matching was applied on a large set of randomly selected grayscale image-pattern pairs under various conditions. For each input image, a pattern of a given size was selected at a random location. To 


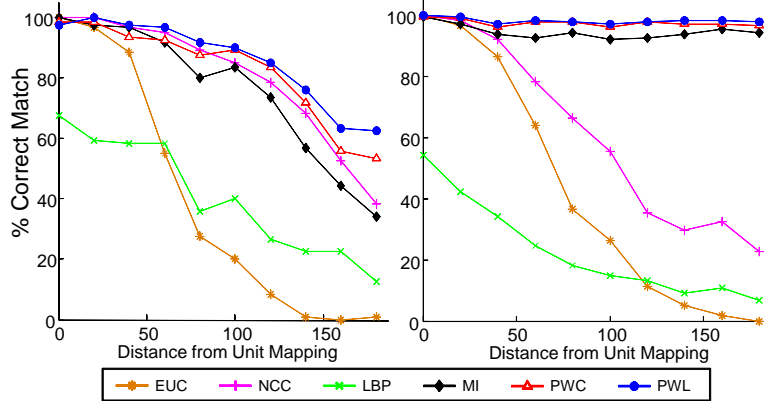

Fig. 5. Pattern detection performance vs. extremity of the tone mapping for (a) monotonic mapping with noise (b) non-monotonic mapping with noise.

avoid "uninteresting" patterns, these locations were selected from amongst the "structured" regions of the image (i.e. locations where the eigen-values of the structured tensor [4] sum above a threshold). Given an image and a selected pattern, a random tone mapping was applied to the image (with additive noise) and the original selected pattern was then sought in the mapped image. Distances were calculated for all possible locations in the tone-mapped image, and the window associated with the minimal distance was considered the matched window. If the matched window was detected at the correct position the match is considered a correct detection (using the top 5 or 10 minimal distance windows, did not significantly change the results).

Sensitivity to Mapping Extremity - Figure 5 displays the detection rate as a function of the extremity of the tone mapping applied to the image. Extremity was measured as the RMS distances between the original range of values $([0 . .255])$ and the mapped tone values. Results are shown separately for monotonic mappings (Figure 5a) and for non-monotonic mappings (Figure $5 \mathrm{~b}$ ). Each data point represents the detection rate (in percentages) over 2000 randomly selected image-pattern pairs. Images were of size $200 \times$ 200 and patterns of size $20 \times 20$. Tone mappings were generated by randomly selecting six new tone values serving as the mapping values for six equally spaced source tone values (in the range $[0 . .255]$ ). The tone mapping was defined as a piecewise linear function passing through the selected values. For monotonic mappings the randomly selected tone values were sorted in increasing order prior to the construction of the tone mapping. Gaussian noise with s.t.d. of 15 gray-values was added to each mapped image before pattern matching was performed.

We note a counter-intuitive observation: monotonicity constrains the possible mappings and typically produces deeply convex or concave functions for extreme mappings. This implies loss of spatial details in image regions which affects pattern detection. Nonmonotonic mappings on the other hand, produce false contours but typically maintain image structure (edges are preserved though possibly with change of

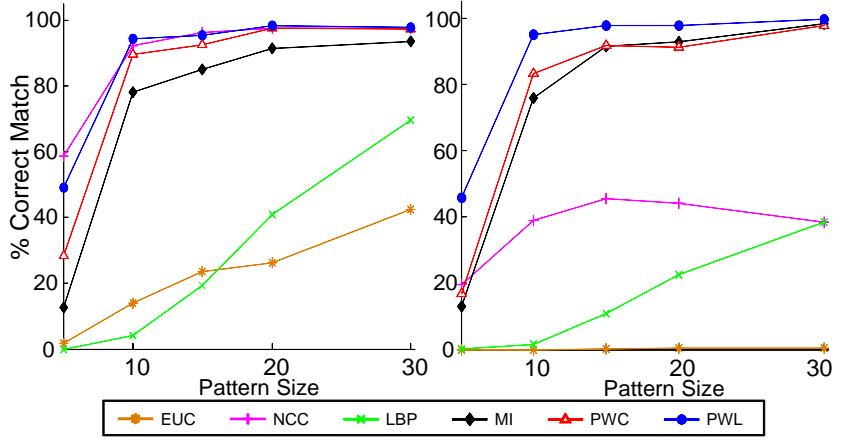

Fig. 6. Performance comparison as a function of pattern size. (a) For specific monotonic mapping. (b) For specific non-monotonic mapping.

contrast). Thus, as will be seen, performance under monotonic mappings is often degraded compared to non-monotonic mappings.

Figure 5 shows that the Euclidean distance and the LBP degrade very fast with mapping extremity. This is expected for the Euclidean case, however, the LBP shows poor performance also in monotonic mappings under which it should perform well. This can be explained by the additive noise to which the LBP is very sensitive, as will be shown below. The NCC is expected to fail in both monotonic and non-monotonic mappings, however in the monotonic case, mapping is smooth and can be approximated locally as linear. Thus, NCC performs relatively well under monotonic mappings compared to the non-monotonic mappings.

It can be seen that the MTM approach in both PWC (unfilled markers) and PWL (solid markers) schemes, performs very well and on par with the MI approach. Both, MTM and MI perform better under non-monotonic mappings than under monotonic mappings due to the observation mentioned above. The MTM and MI methods were optimized for bin size (bin size 40 for MTM and 20 for MI).

Sensitivity to Noise, Pattern Size and Bin SizeWe examined the sustainability of the mentioned distances to additive noise and its performance under various pattern sizes. Figure 6 shows the detection rate for various pattern sizes under a specific monotonic mapping (Figure 6a) and non-monotonic mapping (Figure 6b). All images were contaminated with Gaussian noise with s.t.d. $=15$. It can be seen that for small patterns (under $10 \times 10$ pixels) detection rates are very low in all methods. This behavior stems from the fact that histogram bins of small sized patterns are sparsly populated if at all. This may produce an under-determined system or an over-fitting solution. For this reason techniques using a low number of free parameters are preferable and outperform other methods in small pattern scenarios (NCC for monotonic mappings and MTM-PWL). This phenomena is also shown below in Figure 8.

Figure 7 evaluates the sensitivity of the above methods to additive noise. Pattern matching was 

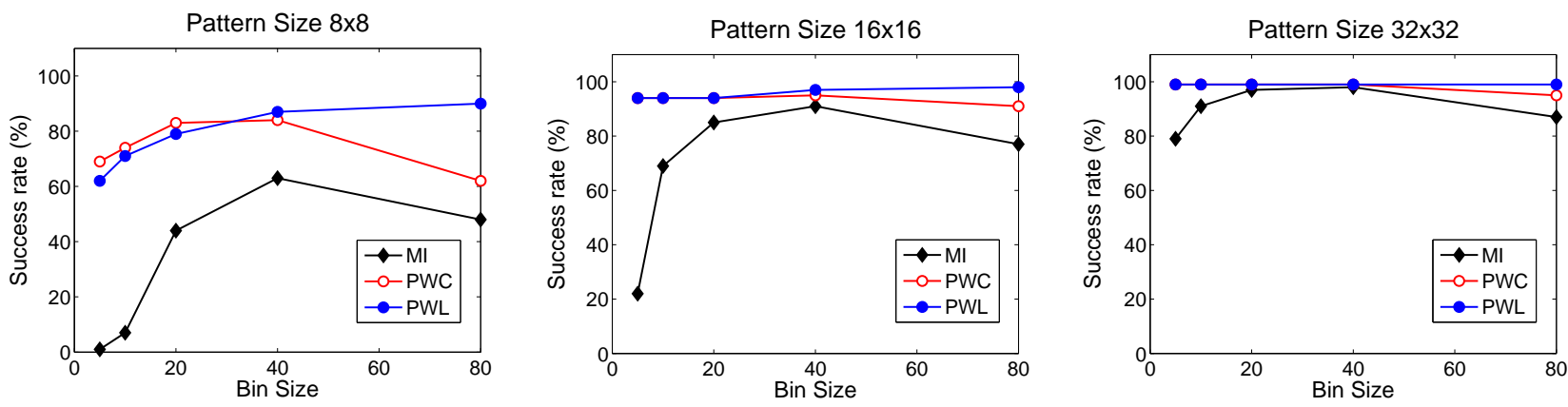

Fig. 8. Comparison of MI and MTM as a function of bin size. For patterns of size $8 \times 8,16 \times 16$ and $32 \times 32$.

performed under a specific mapping with Gaussian noise of varying variance added to each image. As above, data points represent average results over 2000 randomly selected image-pattern pairs. Figures $7 \mathrm{a}$ and $7 \mathrm{~b}$ plot the results for monotonic and non-monotonic mappings respectively. Overall, the results resemble the behavior shown above in Figure 6. Methods with a small number of free parameters perform better, as long as they model well the possible tone-mappings. It can be seen that in both cases MTM-PWL is advantageous over MTM-PWC especially under severe noise conditions.

Finally, we test for the sensitivity of MTM to bin size and compare with that of MI. Figure 8 shows detection rates for MTM-PWC, MTM-PWL and MI over different bin sizes. Results are shown for three different pattern sizes $(8 \times 8,16 \times 16$ and $32 \times 32)$. Each data point is a result of 200 randomly selected image-pattern pairs. For every image-pattern pair, a random monotonic mapping was generated, within the extremity range of 40-60, and Gaussian noise (s.t.d. $=20$ ) was added. These plots show the difference in sensitivity to bin-size between the approaches. As expected, MTM-PWL outperforms MTM-PWC accross pattern sizes as well as MI, and is especially advantageous when using large bin size on smaller patterns. MI shows larger sensitivity to bin size with decrease in performance for smaller bin sizes.

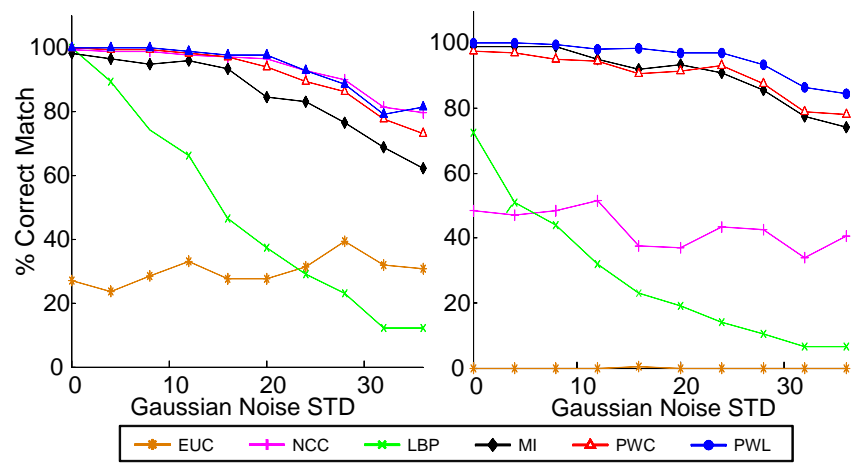

Fig. 7. Performance comparison as a function of added noise. (a) For specific monotonic mapping. (b) For specific non-monotonic mapping.

\subsection{Run Time}

A significant advantage of MTM over MI is computational efficiency. Figure 9 displays run times of pattern matching using different schemes under varying pattern sizes. For MI and MTM, run times are shown for different bin sizes as well. Run times shown are the average over 10 runs. Run time was measured on an Intel $1.70 \mathrm{GHz}$ Pentium M. Since MI requires the computation of the joint histogram for every image window pair, it is more computationally demanding than MTM and other approaches. Furthermore, run time for MI increases with the number of bins. On the other hand, run time of the MTM-PWC scheme is on the order of a single image convolution (Section 4.1) and thus on par with the NCC and Euclidean approaches. Run time of the MTM-PWL scheme is slightly higher than the MTM-PWC (two image convolutions). The size of bins in both, MTM-PWC and MTM-PWL, has very little effect on the run time.

\subsection{Results on Real Images - MTM vs. NCC and MI}

In real scenarios, non-linear mappings between images commonly occur due to differences in camera settings (e.g. gamma correction, white balancing etc.). However, in such cases, local monotonicity is typically maintained and NCC often performs very well. The cases of interest in terms of this work are cases in which NCC is challenged by strong non-monotonicity of the tone mappings. In such cases MTM forms a natural generalization to NCC in terms of performance

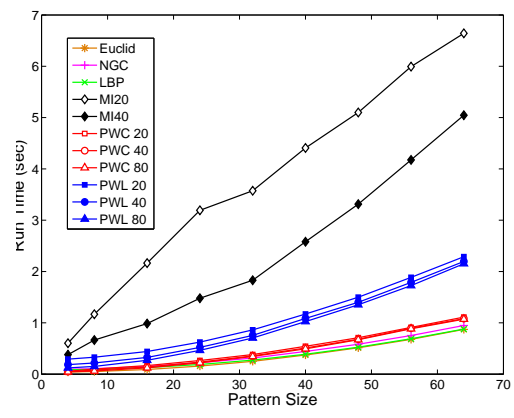

Fig. 9. Run time of various pattern matching schemes (with different bin sizes) as function of pattern sizes. 

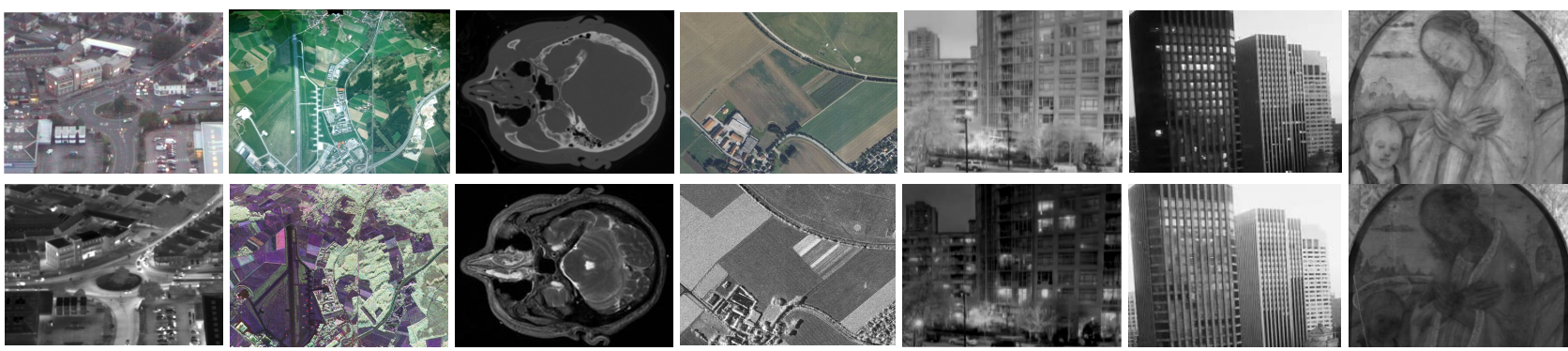

Fig. 10. Examples of image pairs (top-bottom) used in experiments. Pairs are (left to right): visual-IR, visual-SAR, CT-MRI, Visual-SAR, illumination changes, 2 bands of hyperspectral image (SWIR range).

and run times. These cases of interest arise when a scene is captured under very different illumination conditions introducing cast shadows and highlights. Another source of non-monotonicity is when a pair of images are acquired using different modality cameras, such as: visual, SAR, IR, CT, MRI. Such pairs often do not match under global tone mapping but are locally compatible under a non-linear tone mapping.

We compare the performance of MTM under these cases. A collection of pairs of images of the types described above were used. For every pair a pattern was randomly selected in one image and sought in the other. Note that although illumination, highlights and shadows are often revealed only in sub regions of the image, we did not restrict pattern selection to those regions alone.

Examples of pairs of images from our collection are shown in Figure 10. Figure 11 shows 2 examples displaying detection rates of pattern matching between pairs of images for various pattern sizes. The recorded performance is an average over 100 (randomly selected) patterns for each pattern size. Figure 11a shows rates for a multi-modal image pair (SAR vs. Visual - Figure 10, $2^{\text {nd }}$ column from left). Figure $11 \mathrm{~b}$ shows rates for an image pair under different illumination (Figure 10, $3^{\text {rd }}$ column from right).

Although the behaviour of MTM, NCC and MI on real images vary greatly between images, performance comparison over many pairs demonstrates a consistent trend as shown in Figure 12. Bars represent improvement of performance of MTM over NCC (left)
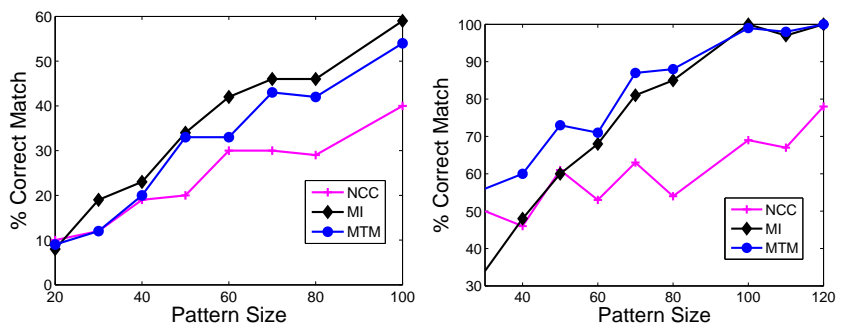

Fig. 11. Performance (\% correct detection) of MTM vs NCC and MI in real images. a) For multimodal image pair b) For image pair under different illumination. Every point is the average over 100 examples. and MI (right). Each bar represents the performance of a particular image pair. Results were averaged over different pattern sizes (ranging from 10-120 pixels squared) where 100 patterns where randomly selected at each size. The first 5 bars are associated with multimodal image pairs and the remaining 4 bars are from image pairs differing in illumination and from multispectral pairs (of proximate spectral bands). This difference can be explained in that images differing in illumination and close-band spectral image pairs have aligned edges. This is not the case in multi-modal pairs where many edges are misaligned or missing. We emphasize that regardless of performance, MTM always significantly outperforms MI in terms of run time requiring time similar to that of NCC.

\section{Additional Applications}

MTM as a similarity measure can be exploited in numerous applications. The advantage of MTM extends naturally from pattern matching to Multi-modal image registration. Image registration requires a registration method [?] which converges to the correct transformation parameters between the registered images and, independently of the registration method, requires a similarity measure to evaluate quality of registration per any transformation parameter that is tested. Irrespective of the methods, we show that MTM is a good similarity measure for image registration, namely provides a deep local minima around the correct registration parameters. MTM is shown to provide this with performance on par with MI and
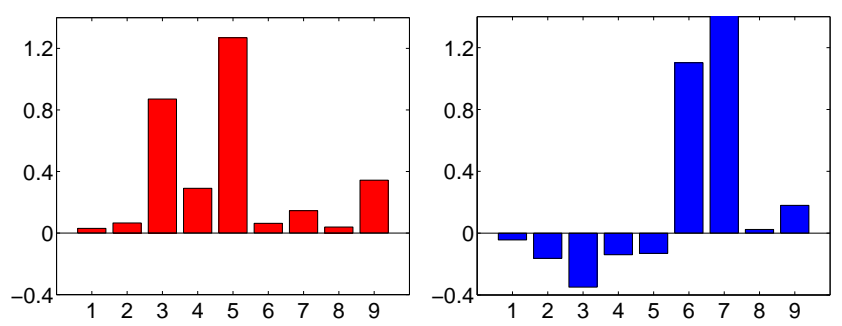

Fig. 12. Histogram of improvement of performance of MTM over NCC (left) and MI (right) for different images. Each bar represents the improvement averaged over 100 examples per each pattern size (10-120 pixels $\left.{ }^{2}\right)$. 
in contrast with poor performance using NCC. To demonstrate this, we evaluated the distance between an image and its modality counterpart under different translation parameters. Figure 13(left) displays distance maps between pairs of images of different modalities (first four pairs in Figure 10). Distance maps for NCC, MI and MTM are shown (left to right). In each map, the center of the map corresponds to the correct translation $(\Delta x=0, \Delta y=0)$ and distance values for other translation parameters (sampled in steps of whole pixels) are represented at the corresponding locations. As can be seen, the minimum distances in the MI and MTM maps correspond to the correct translation with a deep and global minima. The results show that MTM and MI are comparable in their accuracy of alignment whereas NCC largely fails. Similar performance is observed for other multimodal image pairs.

Similar results are obtained when rotation and scale transformations are assumed rather than translation, as shown in Figure 13(right). Rotation parameters (xaxis of map) range from $-90^{\circ}$ to $+90^{\circ}$. Scale parameters (y-axis of map) range from 0.8 to 1.2. As above, the center of the map corresponds to the correct transformation parameters $(\Delta \theta=0, s=1)$.

Note that, evaluating the correct transformation parameters in a multi-modal alignment using MTM, can be implemented very efficiently: image slices need be computed only once for the reference image of the pair, while for the transformed image, only resampling and pointwise multiplication with the image slices is required. In contrast, searching for the transformation parameters using MI requires computing the joint histogram of the image pair for each candidate parameter - a time consuming process.

We briefly mention that MTM has also been exploited to detect shadows in video surveillance sequences [7]. Shadows were distinguished from pedestrians in foreground regions of the video. Shadow removal is difficult in such cases since shadows are non uniform, noisy and tend to have wide penumbras [2]. However, exploiting the assumption that shadow regions are a (not necessarily linear) tone mapping of the background, MTM can be used to evaluate the structural similarity between foreground and corresponding pixels in background image. Small MTM distances relate to shadowed pixels and high values indicate differently structured content, namely, foreground objects (pedestrians). For details see [7].

\section{Discussion and Conclusions}

The MTM and MI approaches are similar in spirit. While MI maximizes the entropy reduction in $\mathbf{w}$ given $\mathbf{p}$, MTM maximizes the variance reduction in $\mathbf{w}$ given $\mathbf{p}$. Both entropy and variance are measures of uncertainty. While variance is a quantitative measure preferring a compact distribution of samples, the

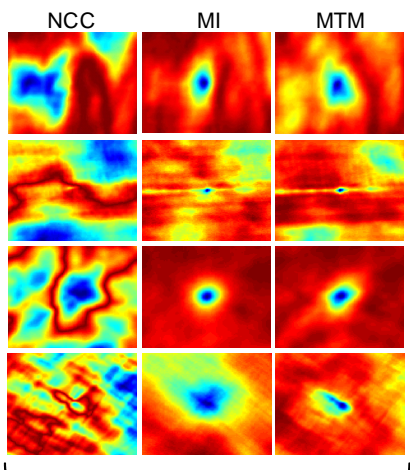

Horizontal + Vertical Translation

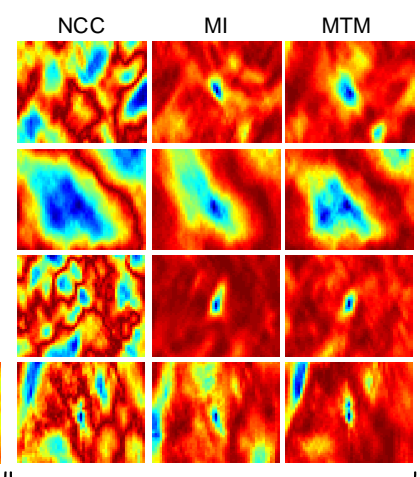

Rotation + Scaling
Fig. 13. Distance maps between multi-modal image pairs for horizontal and vertical translations and for rotation and scale. Image pairs are from Figure 10.

entropy is a qualitative measure disregarding bin rearrangements. The use of variance rather than entropy is critical when a small number of samples are available. Nevertheless, although MTM demonstrates superior performance with respect to run time and stability under sparse samples, it relies on functional dependency between $\mathbf{p}$ and $\mathbf{w}$. When this assumption is violated, e.g. in multi-modal images (between which functional mapping does not necessarily exist), MI often outperforms MTM, although at the expense of longer run time. The following table summarizes the comparison between the MI and the MTM schemes.

\begin{tabular}{|c||c|c|}
\hline & MI & MTM \\
\hline \hline Maximize & entropy reduction & variance reduction \\
\hline Speed & slow & fast \\
\hline Bin size & sensitive & insensitive \\
\hline Measure & qualitative & quantitative \\
\hline
\end{tabular}

In this paper, a fast pattern matching scheme called Matching by Tone Mapping (MTM) was introduced. The distance measure used is expressed as a minimization problem over all possible tone mappings. Thus, by definition, the MTM is invariant to non-linear tone mappings (both monotonic and non-monotonic). Furthermore, MTM is shown to be a generalization of the NCC for non-linear mappings and actually reduces to NCC when mappings are restricted to be linear [27]. An efficient computation of the MTM is proposed requiring computation time similar to the fast implementation of NCC. Considering MTM in terms of a regression scheme, we extend it to higher order regression which allows greater robustness to noise and sparse data.

\section{REFERENCES}

[1] V. Ramesh A. Mittal. An intensity-augmented ordinal measure for visual correspondence. In IEEE Conf. on Computer Vision and Pattern Recognition, pages 849-856, 2006.

[2] E. Arbel and H. Hel-Or. Shadow removal using intensity surfaces and texture anchor points. IEEE Trans. Pattern Analysis and Machine Intelligence, 33(6):1202-1216, 2011. 
[3] C. Barnes, E. Shechtman, A. Finkelstein, and D. Goldman. Patchmatch: a randomized correspondence algorithm for structural image editing. In SIGGRAPH, 2009.

[4] J. Bigun and G. Granlund. Optimal orientation detection of linear symmetry. In 1st IEEE Int. Conf. on Computer Vision, pages 433-438, 1987.

[5] R. Brunelli. Template Matching Techniques in Computer Vision: Theory and Practice. Wiley, 2009.

[6] A. Buades and B. Coll. A non-local algorithm for image denoising. In Proc. IEEE Conf. on Computer Vision and Pattern Recognition, pages 60-65, 2005.

[7] E. Bullkich, I. Ilan, Y. Moshe, Y. Hel-Or, and H. Hel-Or. Moving shadow detection by nonlinear tone-mapping. In Int. Conf. on Systems, Signals and Image Processing (IWSSIP), 2012.

[8] M. Calonder, V. Lepetit, C. Strecha, and P. Fua. Brief: Binary robust independent elementary features. European Conference on Computer Vision, pages 778-792, 2010.

[9] S.D. Conte and C. DeBoor. Elementary Numerical Analysis. McGraw-Hill, New York, 1972.

[10] G.S. Cox. Review: Template matching and measures of match in image processing. University of Cape Town, TR, 1995.

[11] N. Dalal and B. Triggs. Histograms of oriented gradients for human detection. In IEEE Conference on Computer Vision and Pattern Recognition, volume 1, pages 886-893, 2005.

[12] R.O. Duda, P.E. Hart, and D.G. Stork. Pattern classification. John Willey E Sons, 2001.

[13] R.C. Gonzalez and R.E. Woods. Digital Image Processing. Prentice-Hall, 2006.

[14] C.H. Goulden. Methods Of Statistical Analysis. John Wiley and Sons, 1939

[15] C. Harris and M. Stephens. A combined corner and edge detector. In Alvey vision conference, volume 15, page 50, 1988.

[16] H. Hel-Or. Matlab code "http://www.faculty.idc.ac.il/toky/ software/software.htm".

[17] Y. Hel-Or and D. Shaked. A discriminative approach for wavelet denoising. IEEE Trans. on Image Processing, 17(4):443$457,2008$.

[18] N. Jojic, B. J. Frey, and A. Kannan. Epitomic analysis of appearance and shape. In IEEE Int. Conference on Computer Vision, volume 1, pages 34-41, 2003.

[19] J.P. Lewis. Fast normalized cross-correlation. In Vision Interface, pages 120-123, 1995.

[20] S. Kagarlitsky, Y. Moses, and Y. Hel-Or. Piecewise-consistent color mappings of images acquired under various conditions. In The 12th IEEE International Conference on Computer Vision, Kyoto, Japan, Sept 2009.

[21] D.G. Lowe. Distinctive image features from scale-invariant keypoints. International journal of computer vision, 60(2):91-110, 2004

[22] K. Mikolajczyk and C. Schmid. A performance evaluation of local descriptors. IEEE Transactions on Pattern Analysis and Machine Intelligence, 27(10):1615-1630, 2005.

[23] T. Ojala, M. Pietikäinen, and T. Mäenpää. Multiresolution gray-scale and rotation invariant texture classification with local binary patterns. IEEE Trans. Pattern Anal. Mach. Intell., 24(7):971-987, 2002.

[24] F. Porikli. Integral histogram:a fast way to extract histograms in cartesian spaces. In Proc. IEEE Conf. on Computer Vision and Pattern Recognition, pages 829-836, 2005.

[25] A. Roche, G. Malandain, X. Pennec, and N. Ayache. The correlation ratio as a new similarity measure for multimodal image registration. Lecture Notes in Computer Science, 1496:1115-1124, 1998.

[26] A. Roche, G. Malandain, X. Pennec, and N. Ayache. Multimodal image registration by maximization of the correlation ratio. Technical Report 3378, Institut National De Recherche En Informatique Et En Automatique, 1998.

[27] S. Ross. A First Course in Probability. Prentice Hall, 1998.

[28] D. Simakov, Y. Caspi, E. Shechtman, and M. Irani. Summarizing visual data using bidirectional similarity. In IEEE International Conference on Computer Vision and Pattern Recognition, pages $1-8,2008$.

[29] T. Hastie and R. Tibshirani and J.H. Friedman. The Elements of Statistical Learning. Springer, 2003.

[30] P. Viola and W.M. Wells. Alignment by maximization of mutual information. International journal of computer vision, 24(2):137-154, 1977.
[31] Y. Wei and L. Tao. Efficient histogram-based sliding window. In Proc. IEEE Conf. on Computer Vision and Pattern Recognition, pages 3003-3010, 2010.

[32] R. Zabih and J. Woodfill. Non-parametric local transforms for computing visual correspondence. In European Conference on Computer Vision, pages 151-158. Springer-Verlag, 1994.

[33] M. Zibulevski. Code at "http://ie.technion.ac.il/ mcib".

\section{APPENDIX A}

We solve for $\hat{\beta}$ in the system $G \hat{\beta}=Q^{T} \mathbf{w}$ where $Q=$ $Q(\mathbf{p})$ is the SLT-PWL matrix of $\mathbf{p}$ and $G=Q^{T} Q$ is a symmetric tridiagonal matrix, with main diagonal entries:

$$
\varphi_{p}^{j}=\overline{\mathbf{p}}^{j} \cdot \overline{\mathbf{p}}^{j}=\sum_{i \in g_{\mathbf{p}}^{j}}\left(1-r\left(p_{i}\right)\right)^{2}+\sum_{i \in g_{\mathbf{p}}^{j-1}}\left(r\left(p_{i}\right)\right)^{2}, \quad j=1 \cdot \cdot k+1
$$

and the off diagonal entries:

$$
\psi_{p}^{j}=\overline{\mathbf{p}}^{j} \cdot \overline{\mathbf{p}}^{j+1}=\sum_{i \in g_{\mathbf{p}}^{j}}\left(1-r\left(p_{i}\right)\right) r\left(p_{i}\right), \quad j=1 \cdots k
$$

Defining $\rho_{j}=\mathbf{w} \cdot \overline{\mathbf{p}}^{j}$ we have:

$$
\underbrace{\left[\begin{array}{ccccc}
\varphi_{p}^{1} & \psi_{p}^{1} & & & 0 \\
\psi_{p}^{1} & \varphi_{p}^{2} & \psi_{p}^{2} & & \\
& \psi_{p}^{2} & \varphi_{p}^{3} & \ddots & \\
& & \ddots & \ddots & \psi_{p}^{k} \\
0 & & & \psi_{p}^{k} & \varphi_{p}^{k+1}
\end{array}\right]}_{G(\mathbf{p})=Q^{T} Q} \cdot \underbrace{\left[\begin{array}{c}
\hat{\beta}_{1} \\
\hat{\beta}_{2} \\
\hat{\beta}_{3} \\
\vdots \\
\hat{\beta}_{k+1}
\end{array}\right]}_{\hat{\beta}}=\underbrace{\left[\begin{array}{c}
\rho_{1} \\
\rho_{2} \\
\rho_{3} \\
\vdots \\
\rho_{k+1}
\end{array}\right]}_{Q(\mathbf{p})^{T} \mathbf{w}}
$$

Since $G$ is tridiagonal, this linear system can be solved with a linear number of operations using a simplified version of the Gaussian elimination method [9]. The process involves a forward sweep that eliminates the $\psi_{p}^{i}$ 's below the main diagonal, followed by a backward substitution that produces the solution.

In the first step the above system is modified to a new set of equations using Gaussian elimination:

$$
\left[\begin{array}{ccccc}
1 & \omega_{p}^{1} & & & 0 \\
0 & 1 & \omega_{p}^{2} & & \\
& 0 & 1 & \ddots & \\
& & \ddots & \ddots & \omega_{p}^{k} \\
& & & 0 & 1
\end{array}\right] \cdot\left[\begin{array}{c}
\hat{\beta}_{1} \\
\hat{\beta}_{2} \\
\hat{\beta}_{3} \\
\vdots \\
\hat{\beta}_{k+1}
\end{array}\right]=\left[\begin{array}{c}
\sigma_{1} \\
\sigma_{2} \\
\sigma_{3} \\
\vdots \\
\sigma_{k+1}
\end{array}\right]
$$

where the new coefficients are calculated as follows:

$$
\omega_{p}^{i}= \begin{cases}\frac{\psi_{p}^{1}}{\varphi_{p}^{1}} & \text { for } \quad i=1 \\ \frac{\psi_{p}^{i}}{\varphi_{p}^{i}-\omega_{p}^{i-1} \psi_{p}^{i-1}} & \text { for } \quad i=2,3, \ldots, k\end{cases}
$$

and

$$
\sigma_{i}= \begin{cases}\frac{\rho_{i}}{\varphi_{p}^{1}} & \text { for } \quad i=1 \\ \frac{\rho_{i}-\sigma_{i-1} \psi_{p}^{i-1}}{\varphi_{p}^{i}-\omega_{p}^{i-1} \psi_{p}^{i-1}} & \text { for } \quad i=2,3, \ldots, k+1\end{cases}
$$


The solution is then obtained using backward substitution:

$$
\begin{aligned}
\hat{\beta}_{k+1} & =\sigma_{k+1} \\
\hat{\beta}_{i} & =\sigma_{i}-\omega_{p}^{i} \hat{\beta}_{i+1}, \quad \text { for } i=k, k-1, \cdots, 1(28)
\end{aligned}
$$

Note, that during the elimination step the coefficients $\left\{\omega_{i}\right\}$ are calculated only once for all candidate windows, while $\left\{\sigma_{i}\right\}$ and $\hat{\beta}$ must be calculated for each window. Since $Q^{T} \mathbf{w}$ is calculated using $O(m)$ operations (Section 4) and calculating $\hat{\beta}$ requires an additional $O(k)$ operations the entire process requires $O(m+k)$ operations. 